\title{
MORTALIDAD PERINATAL E INFANTIL Y EL BAJ O PESO AL NACER EN CHILE Y CUBA EN EL PERIODO 1991-2001
}

\author{
Enrique Donoso S. ${ }^{1}$, Andrés Donoso D. ${ }^{a}$, Luis Villarroel del P., \\ ${ }^{1}$ Departamento de Obstetricia y Ginecología, ${ }^{2}$ Departamento de Salud Pública, Facultad de Medicina, Pontificia \\ Universidad Católica de Chile
}

a Interno de Medicina, Universidad Católica de Chile.

b Estadístico

\section{RESUMEN}

Objetivo. Comparar entre Chile y Cuba, países con diferentes sistemas de salud y realidades socioeconómicas, los indicadores de morbimortalidad perinatal e infantil. Material y método. En el período 1991-2001, se comparan entre Chile y Cuba, las tasas de mortalidad fetal tardía, neonatal precoz, perinatal e infantil y el bajo peso al nacer. La tendencia de las variables se estudia mediante análisis de correlación de Pearson. Los promedios y las causas de muerte se analizan según t de Student y Chi cuadrado, respectivamente. La información se obtuvo de las bases de estadísticas vitales de Chile y Cuba. Resultados. Cuba tuvo tasas de mortalidad neonatal precoz e infantil significativamente menores que Chile y mayores de mortalidad fetal tardía y perinatal y de bajo peso al nacer. En Chile, la tendencia descendente de la mortalidad fetal tardía, neonatal precoz, perinatal e infantil fue significativa; similar tendencia presentó en Cuba la mortalidad neonatal precoz, perinatal e infantil y el bajo peso al nacer. La mortalidad fetal tardía en Cuba y el bajo peso al nacer en Chile, no presentaron cambios significativos. En Cuba, la mortalidad infantil por afecciones perinatales, malformaciones congénitas y neumonía fueron significativamente menores que en Chile y mayores por accidentes y sepsis. Conclusiones. Chile y Cuba, en el período 1991-2001, redujeron significativamente la mortalidad perinatal e infantil, de acuerdo a sus realidades históricas, sociales, económicas y principios éticos vigentes.

\section{KEY WORDS: Mortalidad perinatal, mortalidad infantil, bajo peso al nacer, estadísticas vitales latinoamericanas}

\section{SUMMARY}

Objective. To compare perinatal and infant mortality rate and low birth weight between Chile and Cuba. Method. Fetal, early neonatal, perinatal and infant mortality rate for Chile and Cuba for the period between 1991 to 2001 were analyzed. The information was obtained from the National Vital Statistical Records from the respective countries. Pearson correlation, $\mathrm{T}$ student and Chi square test were used for comparison. Results. Early neonatal and infant mortality rate were significantly lower for Cuba compared with Chile. Late fetal mortality rate, perinatal mortality rate and low birth weight were significantly lower for Chile compared with Cuba. There was a similar trend to decrease in all the indices analyzed for both countries. Infant mortality in Cuba was more related to accidents and sepsis and less due to perinatal factors, congenital anomalies, pneumonia or congenital anomalies compared to Chile. Conclusion. Both Chile and Cuba showed a significant reduction in perinatal and infant mortality for the period between 1991 to 2001 .

KEY WORDS. Perinatal mortality, infant mortality, low birth weight, latinamerican vital statistics 


\section{INTRODUCCIÓN}

La mortalidad infantil es un indicador biodemográfico que refleja el grado de desarrollo social, económico y sanitario de un país. El proceso reproductivo a través de la prematurez, la restricción del crecimiento intrauterino, las infecciones perinatales y las malformaciones congénitas incide directamente en la mortalidad perinatal e infantil y el bajo peso al nacer.

Las diferencias sociales y económicas entre los países determinan una gran desigualdad en mortalidad infantil (1). En la Región de las Américas la mortalidad infantil fluctúa entre 6 y 116/ 1000 nacidos vivos, rango dado por Canadá y Haití, respectivamente (2).

En 1960, la mortalidad infantil en Chile y Cuba eran respectivamente de 119,5 y $35,9 / 1000$ nacidos vivos $(3,4)$, las circunstancias históricas hicieron que desarrollaran sistemas de salud que les permitió liderar los indicadores sanitarios en Latinoamérica (5). Así en 2001, Chile y Cuba redujeron respectivamente la mortalidad infantil a 8,3 y $6,2 / 1000$ nacidos vivos $(6,7)$. Los sistemas de salud de esos países son diferentes en cobertura, infraestructura y presupuesto; el cubano es $100 \%$ estatal, con 530 médicos/100.000 habitantes y un gasto en salud per cápita de 186 dólares internacionales, mientras que el chileno es $66,5 \%$ estatal, con 110 médicos/100.000 habitantes y un gasto en salud per cápita de 697 dólares internacionales $(8,9)$. Ambos países cuentan con una infraestructura para la atención maternal que les permite una amplia cobertura del control prenatal y atención profesional del parto cercano al $100 \%$ (5).

El objetivo de este estudio es comparar entre Chile y Cuba en el período 1991-2001, las tasas y tendencias de la mortalidad perinatal e infantil, del bajo peso al nacer y las causas de muerte en el primer año de vida.

\section{MATERIAL Y MÉTODO}

Se compara entre Chile y Cuba, en el período 1991-2001, las tasas y tendencias de la mortalidad perinatal (mortalidad fetal tardía y neonatal precoz) e infantil y el bajo peso al nacer, además se analizan las causas de muerte infantil ocurridas en 2001. La información se extrae desde las bases de datos de estadísticas vitales de los países estudiados $(6,7)$. Los conceptos de nacido vivo (nv), bajo peso al nacer y de mortalidad perinatal, fetal tardía, neonatal precoz e infantil, son los internacionalmente aceptados. El análisis comparativo entre las principales causas de mortalidad infantil en 2001, se efectuó según información establecida en la base de datos de Cuba a la que se adecuó la de Chile $(6,7)$. El estudio de tendencias se efectuó según análisis de correlación de Pearson, la comparación de proporciones mediante Chi cuadrado y la comparación de promedios con $\mathrm{t}$ de Student para muestras independientes. Las fluctuaciones de los parámetros se presentan según porcentaje de cambio entre el valor de la variable en 2001 comparado con 1991. Se estableció un nivel de significación estadística de $p<0,05$.

\section{RESULTADOS}

En el período 1991-2001, Chile presentó tasas promedio de mortalidad fetal tardía $(\mathrm{t}=23,7$; $p<0,001)$, mortalidad perinatal $(t=7,1 ; p<0,001)$ y de bajo peso al nacer $(t=7,1 ; p<0,001)$ significativamente menores que Cuba; mientras que Cuba tuvo tasas promedio de mortalidad neonatal pre$\operatorname{coz}(t=-4,4 ; p<0,001)$ e infantil $(t=-3,7 ; p=0,001)$ significativamente menores que Chile (Tabla I).

En Cuba, los nacimientos $(R=-0,701 ; p=0,016)$, el bajo peso al nacer $(R=-0,886 ; p<0,001)$ y las tasas de mortalidad neonatal precoz $(R=-0,946$; $p<0,001)$, perinatal $(R=-0,919 ; p<0,001)$ e infantil

Tabla I

COMPARACIÓN DE LAS TASAS PROMEDIO DE MORTALIDAD FETAL TARDÍA, NEONATAL PRECOZ, PERINATAL E INFANTIL Y DE BAJO PESO AL NACER EN CUBA Y CHILE, PARA EL PERÍODO 1991-2001

\begin{tabular}{lrc}
\hline Tasas & Cuba & Chile \\
& $\bar{x} \pm D E$ & $\bar{x} \pm D E$ \\
\hline Mortalidad fetal tardía (x 1000 nv) & $9,5 \pm 0,2$ & $4,7 \pm 0,5$ \\
Mortalidad neonatal precoz (x 1000 nv) & $3,5 \pm 0,7$ & $4,9 \pm 0,7$ \\
Mortalidad perinatal (x 1000 nv)* & $13,0 \pm 0,8$ & $9,7 \pm 1,3$ \\
Mortalidad infantil (x 1000 nv) & $8,3 \pm 1,6$ & $11,2 \pm 2,0$ \\
Bajo peso al nacer (x 100 nv) & $7,4 \pm 1,1$ & $5,0 \pm 0,2$ \\
\hline
\end{tabular}

t de Student: *Diferencia significativa. 
$(R=-0,954 ; p<0,001)$ presentaron una tendencia descendente significativa, mientras que la tasa de mortalidad fetal tardía $(R=-0,588 ; p=0,057)$ no presentó cambios significativos (Tabla II). En Chile, los nacimientos $(R=-0,995 ; p<0,001)$ y las tasas de mortalidad fetal tardía $(R=-0,756 ; p=0,007)$, neonatal precoz $(R=-, 924 ; p<0,001)$, perinatal $(R=-$ $0,901 ; p<0,001)$ e infantil $(R=-0,973 ; p<0,001)$ presentaron una tendencia descendente significativa, mientras que el bajo peso al nacer $(R=-0,217$; $\mathrm{p}=0,522$ ) no presentó cambios significativos (Tabla
III). Los dos países redujeron la mortalidad infantil y neonatal precoz sobre el $40 \%$, mientras que la variable con menor reducción en Chile fue el bajo peso al nacer y en Cuba la mortalidad fetal tardía (Tabla IV).

En 2001, la mortalidad infantil en Cuba fue significativamente menor a la de Chile $\left(\chi^{2}=53,9\right.$; $p<0,001)$ y las muertes infantiles dependientes del proceso reproductivo (P00 - P99, Q00 - Q99) determinaron el $73,4 \%$ de las muertes infantiles en Cuba y el $70,4 \%$ en Chile (Tabla V). La mortalidad

Tabla II

TENDENCIA DE LA NATALIDAD (NV) Y DE LAS TASAS DE MORTALIDAD FETAL TARDÍA (TMFT), NEONATAL PRECOZ (TMNP), PERINATAL (TMPN) E INFANTIL (TMI) Y DEL BAJO PESO AL NACER (TBPN) EN CUBA, 1991-2001

\begin{tabular}{ccccccc}
\hline Año & $\begin{array}{c}n v^{* *} \\
(n)\end{array}$ & $\begin{array}{c}T B P N^{* *} \\
(x \quad 100 n v)\end{array}$ & $\begin{array}{c}T M F T^{*} \\
(x \quad 1000 n v)\end{array}$ & $\begin{array}{c}T M N P^{* *} \\
(x \quad 1000 n v)\end{array}$ & $\begin{array}{c}T M P N^{* *} \\
(x \quad 1000 n v)\end{array}$ & $\begin{array}{c}T M I^{* *} \\
(x \quad 1000 n v)\end{array}$ \\
\hline 1991 & 173.896 & 7,8 & 9,7 & 4,6 & 14,3 & 10,7 \\
1992 & 157.349 & 8,6 & 9,6 & 4,6 & 14,2 & 10,2 \\
1993 & 152.233 & 9,0 & 9,8 & 4,0 & 13,8 & 9,4 \\
1994 & 147.265 & 8,9 & 9,7 & 4,0 & 13,7 & 9,9 \\
1995 & 147.170 & 7,9 & 9,6 & 3,9 & 13,5 & 9,4 \\
1996 & 140.276 & 7,3 & 9,2 & 3,2 & 12,4 & 7,9 \\
1997 & 152.681 & 6,9 & 9,5 & 3,0 & 12,5 & 7,2 \\
1998 & 151.080 & 6,7 & 9,5 & 2,9 & 12,4 & 7,1 \\
1999 & 150.785 & 6,5 & 9,3 & 2,9 & 12,2 & 6,5 \\
2000 & 143.528 & 6,1 & 9,7 & 3,1 & 12,8 & 7,2 \\
2001 & 138.718 & 5,9 & 9,2 & 2,6 & 11,8 & 6,2 \\
\hline
\end{tabular}

Correlación de Pearson: *No significativa, **Significativa.

Fuente: Anuario Estadístico de Salud de Cuba (7).

Tabla III

TENDENCIA DE LA NATALIDAD (NV) Y DE LAS TASAS DE MORTALIDAD FETAL TARDÍA (TMFT), NEONATAL PRECOZ (TMNP), PERINATAL (TMPN) E INFANTIL (TMI) Y DEL BAJO PESO AL NACER (TBPN) EN CHILE, 1991 - 2001

\begin{tabular}{|c|c|c|c|c|c|c|}
\hline Año & $\begin{array}{l}n V^{* *} \\
(n)\end{array}$ & $\begin{array}{c}\text { TBPN* } \\
(\times 100 n v)\end{array}$ & $\begin{array}{c}\text { TMFT }^{* *} \\
(x 1000 n v)\end{array}$ & $\begin{array}{c}T_{M N P^{* *}} \\
(x 1000 n v)\end{array}$ & $\begin{array}{c}T_{M P N^{* *}} \\
(x 1000 n v)\end{array}$ & $\begin{array}{c}T M I^{* *} \\
(\times 1000 \mathrm{nv})\end{array}$ \\
\hline 1991 & 299.456 & 5,4 & 5,9 & 6,4 & 12,3 & 14,6 \\
\hline 1992 & 293.787 & 5,3 & 5,7 & 6,1 & 11,8 & 14,3 \\
\hline 1993 & 290.438 & 5,0 & 5,3 & 5,3 & 10,6 & 13,1 \\
\hline 1994 & 288.175 & 4,7 & 4,6 & 5,2 & 9,8 & 12,0 \\
\hline 1995 & 279.928 & 4,7 & 4,6 & 4,6 & 9,2 & 11,1 \\
\hline 1996 & 278.729 & 4,8 & 4,5 & 4,8 & 9,3 & 11,1 \\
\hline 1997 & 273.641 & 4,8 & 4,5 & 4,5 & 9,0 & 10,0 \\
\hline 1998 & 270.637 & 5,1 & 4,3 & 4,6 & 8,9 & 10,3 \\
\hline 1999 & 263.867 & 5,2 & 4,1 & 4,5 & 8,6 & 10,1 \\
\hline 2000 & 261.993 & 5,0 & 4,3 & 4,4 & 8,7 & 8,9 \\
\hline 2001 & 259.069 & 5,0 & 4,9 & 3,7 & 8,6 & 8,3 \\
\hline
\end{tabular}

Correlación de Pearson: *No significativa, ** Significativa.

Fuente: Instituto Nacional de Estadísticas de Chile (6). 
Tabla IV

PORCENTAJE DE CAMBIO DE LA NATALIDAD, DEL BAJO PESO AL NACER, DE LA MORTALIDAD FETAL TARDÍA, NEONATAL PRECOZ, PERINATAL E INFANTIL DE CHILE Y CUBA, ENTRE EL AÑO 1991 Y 2001

\begin{tabular}{lcr}
\hline Variable & $\begin{array}{c}\text { Chile } \\
(\%)\end{array}$ & $\begin{array}{r}\text { Cuba } \\
(\%)\end{array}$ \\
\hline Nacidos vivos & $-13,5$ & $-20,2$ \\
Mortalidad fetal tardía & $-17,0$ & $-5,2$ \\
Mortalidad neonatal precoz & $-42,2$ & $-43,5$ \\
Mortalidad perinatal & $-30,1$ & $-17,5$ \\
Mortalidad infantil & $-43,2$ & $-42,1$ \\
Bajo peso al nacer & $-7,4$ & $-24,4$ \\
\hline
\end{tabular}

infantil en Chile por afecciones perinatales $\left(\chi^{2}=4,29\right.$; $p=0,038$ ), malformaciones congénitas y genopatías $\left(\chi^{2}=33,11 ; p<0,001\right)$ e infecciones respiratorias $\left(\chi^{2}=32,2 ; p<0,001\right)$ fue significativamente mayor que en Cuba; mientras que la mortalidad infantil en Cuba por accidentes $\left(\chi^{2}=17,42 ; p<0,001\right)$ y sepsis $\left(\chi^{2}=7,01 ; p=0,008\right)$ fue significativamente mayor que en Chile (Tabla $V$ ).

\section{DISCUSIÓN}

Chile y Cuba en el período 1991-2001, redujeron significativamente la mortalidad perinatal e infantil. Al término del período, la mortalidad perinatal e infantil en Chile fue de 8,6 y $8,3 / 1000 \mathrm{nv}$ y en Cuba de 11,8 y 6,2/1000 nv, cercanas a las de EEUU, país desarrollado de la Región, que en 2001 fue de 6,9 y 6,8/1000 nv, respectivamente (10).

La mortalidad perinatal en Cuba pese a mostrar una reducción significativa, fue sorprendente- mente alta por una elevada tasa de mortalidad fetal tardía que en 2001 fue 3,5 veces mayor que la neonatal precoz, pese a su organizado programa de salud materno-perinatal (11). Chile redujo significativamente la mortalidad perinatal y la de sus componentes con una relación cercana a 1:1, similar a la de los países desarrollados de la Región $(10,12)$. Las posibles explicaciones para estas diferencias son que Cuba tenga un mayor riesgo obstétrico que Chile, o que el control prenatal no es del todo eficiente en la identificación del feto de alto riesgo. Contrariamente, la mortalidad neonatal precoz en Cuba fue extraordinariamente baja y presentó una significativa reducción, permitiendo así que la mortalidad perinatal tuviera similar tendencia. Las posibles explicaciones son que en Cuba el manejo neonatal sea más eficiente, que la alta mortalidad fetal reduzca el riesgo al período neonatal precoz o que la proporción de fetos malformados o genópatas que nacen vivos sea proporcionalmente baja. En Chile el 9,6\% de la mortalidad fetal tardía y el $32,3 \%$ de la mortalidad neonatal precoz es dependiente de malformaciones congénitas y genopatías $(13,14)$, proporción no modificable por la vía del aborto inducido por ser ilegal en todas sus formas (15).

El bajo peso al nacer es uno de los indicadores de morbilidad perinatal más utilizado, ya que concentra a los recién nacidos de mayor riesgo. En Latinoamérica entre 1990 y 1995 la prevalencia promedio del bajo peso fue de $8,2 \%$, contribuyendo con el $76 \%$ de la mortalidad neonatal (16). Chile en el período estudiado estabilizó el indicador en $5 \%$, probablemente como consecuencia del programa de alimentación materna complementaria y la reducción de la pobreza de $38,6 \%$ en 1990 a 20,6\% en 2000 (17), mientras que

Tabla V

PRINCIPALES CAUSAS DE MORTALIDAD INFANTIL (X 100.000 NV) EN CUBA EN 2001 Y SU HOMOLOGACIÓN EN CHILE

\begin{tabular}{|c|c|c|}
\hline $\begin{array}{l}\text { Factores causales } \\
(\text { CIE-10) }\end{array}$ & $\begin{array}{c}\text { Cuba } \\
\text { (n/tasa) }\end{array}$ & $\begin{array}{c}\text { Chile } \\
\text { (n/tasa) }\end{array}$ \\
\hline Ciertas afecciones originadas en el período perinatal $(\mathrm{P} 00-\mathrm{P} 96)^{*}$ & $399 / 287,6$ & $846 / 326,5$ \\
\hline Malformaciones congénitas, deformidades y anomalías cromosómicas (Q00-Q99)* & $233 / 167,9$ & $673 / 259,7$ \\
\hline Accidentes (V01-X59; Y85-86)* & $32 / 23,1$ & $18 / 6,9$ \\
\hline Influenza y neumonía (J10-J18)* & $30 / 21,6$ & $166 / 64,1$ \\
\hline Sepsis (A40-A41) * & $16 / 11,5$ & $10 / 3,9$ \\
\hline Otras & $151 / 108,9$ & $446 / 172,2$ \\
\hline Total $^{*}$ & $861 / 620,7$ & $2.159 / 833,4$ \\
\hline
\end{tabular}

Chi cuadrado: *Diferencia significativa. 
Cuba redujo significativamente la prevalencia del indicador, producto del programa nacional para la reducción del bajo peso al nacer (18), pero aún con una tasa significativamente mayor que la de Chile.

El control de la fecundidad se asocia a la reducción de la mortalidad infantil (19). En el período analizado, ambos países presentaron una significativa reducción de la natalidad y paralelamente un descenso de la mortalidad perinatal e infantil. Sin embargo, Chile tiene un riesgo mayor de morbimortalidad perinatal e infantil por el significativo aumento de los nacimientos en edades reproductivas extremas $(20,21)$, con tasas de fecundidad de 13,5 nacimientos/1000 mujeres de 40-44 años y 64,1 nacimientos/1000 mujeres de 15-19 años, en comparación a Cuba con 3,0 nacimientos/1000 mujeres de 40-44 años y 52,3 nacimientos/1000 mujeres de 15-19 años (6,7).

El análisis de los factores causales de mortalidad infantil en ambos países muestra que el proceso reproductivo explica al menos el $70 \%$ de los fallecimientos. La mortalidad infantil por afecciones originadas en el período perinatal (P00-P96) es significativamente mayor en Chile y la prematurez en 2001 determinó el 38,1\% de las muertes neonatales precoces (6). La introducción del surfactante artificial ha permitido reducir las muertes neonatales, especialmente en los niños con peso entre 1.000 a 1.499 gramos (22); Cuba como consecuencia del bloqueo económico debió emplear surfactante nacional con éxito relativo $(23,24,25)$.

En Chile, la mortalidad infantil por malformaciones congénitas, deformaciones y anomalías cromosómicas (Q00-Q99), es significativamente mayor (259,7/100.000 nv) que en Cuba (167,9/ $100.000 \mathrm{nv}$ ), la cual es cercana a la de EEUU $(136,9 / 100.000$ nv) (10). Las diferencias con Chile se explicarían por la legalización del aborto inducido en esos países (26). Chile podría modificar la tasa de mortalidad infantil, reduciendo las malformaciones congénitas de mayor prevalencia, como son los defectos de cierre del tubo neural y las cardiopatías congénitas, mediante acciones preventivas y terapéuticas. Informes preliminares señalan que el programa nacional de fortificación de las harinas con ácido fólico, redujo en $42 \%$ la incidencia de anencefalia en el período 2001-2002 (27), mientras que la garantización de un oportuno tratamiento a los recién nacidos cardiópatas, susceptibles de corrección quirúrgica, contribuirá a reducir la mortalidad infantil por esa causa (28).

La mortalidad infantil por patología respiratoria infecciosa (J10-J18) ha tenido en Chile al igual que en Cuba, un significativo descenso desde la implementación de los programas de infecciones respiratorias agudas $(18,29)$. La mortalidad infantil por patología respiratoria es significativamente mayor en Chile, lo que podría explicarse por las marcadas diferencias climáticas que aumentaría la prevalencia de las infecciones respiratorias virales especialmente en el invierno chileno (30).

En Cuba, la mortalidad infantil por sepsis (A40A41) fue significativamente mayor que en Chile. Una de las posibles explicaciones para esta diferencia es que el bloqueo económico impuesto a Cuba, haya impedido un adecuado abastecimiento de antibióticos y otros insumos necesarios para el tratamiento de los cuadros sépticos (31), lo que obligó a una intensa racionalización del uso de antibióticos y quimioterápicos, principalmente en las unidades de terapia intensiva pediátrica (18).

No disponemos de antecedentes que permitan explicar la menor mortalidad infantil en Chile por accidentes.

En conclusión, en el período 1991-2001, Chile y Cuba redujeron significativamente la mortalidad perinatal e infantil de acuerdo a sus realidades históricas, sociales, económicas y principios éticos vigentes.

\section{BIBLIOGRAFIA}

1. Castillo-Salgado C, Loyola E, Roca A. Desigualdades en la mortalidad infantil en la Región de las Américas: Elementos básicos para el análisis. Boletín Epidemiológico/OPS 2001; 22(2): 4-7.

2. Ahmad OB, López AD, Inoue M. Reevaluación de la disminución de la mortalidad infantil. Bulletin of the World Health Organization 2000; 78(10): 1175-91.

3. Taucher E, Jofré I. Mortalidad infantil en Chile: el gran descenso. Rev Méd Chile 1997; 125(10): 1225 35.

4. Riverón RL, Azcuy P. Mortalidad infantil en Cuba 1959-1999. Rev Cubana Pediatr 2001; 73(3): 14357.

5. UNICEF. Latin America and the Caribbean. Statistical data. Disponible en: www.unicef.org/statis/ latinamerica.html. Acceso el 28 de diciembre de 2003.

6. INSTITUTO NACIONAL DE ESTADÍSTICAS. Anuarios de Demografía y de Estadísticas Vitales. Chile, 1991-2001.

7. ANUARIO ESTADÍSTICO DE SALUD. CUBA, 2001. Disponible en: www.sld.cu/servicios/estadisticas/. Acceso el 20 de enero de 2004.

8. WORLD HEALTH ORGANIZATION. Indicadores básicos sanitarios y económicos de los Estados miembros. Disponible en: www.who.int/country/en/ index.html. Acceso el 20 de enero de 2004. 
9. MINISTERIO DE PLANIFICACIÓN. Situación de Salud, 2000. Informe Ejecutivo. Santiago de Chile. Agosto de 2001. Disponible en: www.mideplan.cl. Acceso el 28 de diciembre de 2003.

10. Kochanek KD, Martin JA. CDC. Supplemental analyses of recent trends in infant mortality. Disponible en: www.cdc.gov/nchs/about/major/dvs/ mortdata.htm. Acceso: 18 de febrero de 2004.

11. Cardoso UF. El enfoque de riesgo en la atención maternoinfantil: la experiencia de Cuba. En: López G, Yunes Y, Solís JA, Omran AR (eds). Salud Reproductiva de las Américas. Cap 4. OPS/OMS, 1992; 80-95.

12. Arbuckle T, Dzakpasu S, Liu S, Rouleau J, Rusen ID, Turner L, Wu Wen S. Canadian Perinatal Health Report, 2000. Disponible en: www.hc-sc.gc.ca/hpb/ Icdc/brch/reprod.html. Acceso el 20 de enero de 2004.

13. Donoso E, Oyarzún E, Villarroel L. Mortalidad fetal tardía. Chile 1990-1996. Rev Chil Obstet Ginecol 1999; 64(1): 21-28.

14. Donoso E, Villarroel L. Mortalidad neonatal precoz. Chile 1991-1997. Rev Chil Obstet Ginecol 1999; 64(4): 286-291.

15. CÓDIGO SANITARIO. Libro V. Artículo 119. Ley $\mathrm{N}^{\circ}$ 18.826 del 15 de septiembre de 1989.

16. Schwarcz R, Fescina R. Bajo peso al nacer y mortalidad neonatal en América Latina. En: Pérez A, Donoso E (eds). Obstetricia. 3a ed. Cap 62. Santiago, Chile: Edit Mediterráneo 1999; 887-90.

17. MINISTERIO DE PLANIFICACIÓN DE CHILE. Pobreza e indigencia e impacto del gasto social en la calidad de vida. Santiago de Chile, Julio 2001. Disponible en: www.mideplan.cl. Acceso el 28 de diciembre de 2003.

18. Riverón RL. Estrategias para reducir la mortalidad infantil, Cuba 1959-1999. Rev Cubana Pediatr 2000; 72(3): 147-64.

19. THE ALAN GUTTMACHER INSTITUTE. Pregnancy \& Birth. Family planning can reduce high infant mortality levels. Issues in Brief. 2002 Series, № 2. Disponible en: www.guttmacher.org. Acceso el 19 de febrero de 2004.

20. Donoso E, Becker J, Villarroel L. Evolución de la natalidad y del riesgo reproductivo en mujeres de 40 o más años en la década de los 90. REV CHIL OBstet GineCOL 2002, 67(2): 139-42.

21. Donoso E, Becker J, Villarroel L. Natalidad y riesgo reproductivo en adolescentes de Chile, 1990-1999. Rev Panam Salud Pública 2003; 14(1): 3-8.

22. Oto MA, Henríquez MT, Martínez V, Náquira N. Quince años de mortalidad neonatal en un hospital de la Región Metropolitana. Rev Chil Pediatr 2000; 71(1): 12-16.

23. Manzanares D, Fernández R, Moreno O. Un surfactante natural para el tratamiento del síndrome del distress respiratorio neonatal. Rev Cubana Pediatr 1997; 69(1): 3-10.

24. Moreno O, Lee M, Domínguez F, Pascual MA, Alonso A, Giménez G, Manzanares D. Estudio de la eficacia del Surfacén en el distress respiratorio del recién nacido. Rev Cubana Pediatr 1999; 71(2): 60- 71.

25. Acosta R, Arronte J, Cabrera N. Evaluación del Surfacén en el síndrome de dificultad respiratoria del prematuro. Rev Cubana Pediatr 2000; 72(4): 287-94.

26. David H, Pick de Weiss S. El aborto en las Américas. En: López G, Yunes Y, Solís JA, Omran AR (eds). Salud Reproductiva de las Américas. Cap 13. OPS/ OMS 1992; 353-86.

27. INSTITUTO DE NUTRICIÓN Y TECNOLOGÍA DE LOS ALIMENTOS (INTA). Las propiedades preventivas del ácido fólico. Disponible en: www.inta.cl/ noticias/acido-folico/. Acceso 18 de febrero de 2004.

28. MINISTERIO DE SALUD PÚBLICA DE CHILE. Manual AUGE, 2003. Disponible en: www.minsal.cl/ici/ doc-reforma/Manual\%20AUGE\%202003.pdf. Acceso: 15 de febrero de 2004.

29. Guirardi G, Astudillo P, Zúñiga F. El programa IRA en Chile: hitos e historia. Rev Chil Pediatr 2001; 72(4): 292-300.

30. Avendaño L, Céspedes A, Stecher X, Palomino MA. Influencia de virus respiratorios, frío y contaminación aérea en la infección respiratoria aguda baja del lactante. Rev Méd Chile 1999; 127(9): 1073-78.

31. Barry M. Effect of the U.S. embargo and economic decline on health in Cuba. Ann Intern Med 2000; 132: $151-54$. 